\title{
BMJ Open Self-reported health without clinically measurable benefits among adult users of multivitamin and multimineral supplements: a cross-sectional study
}

\author{
Manish D Paranjpe (D , ${ }^{1}$ Alfred C Chin, ${ }^{2}$ Ishan Paranjpe, ${ }^{3}$ Nicholas J Reid, ${ }^{4}$ \\ Phan Q Duy, ${ }^{5}$ Jason K Wang, ${ }^{1}$ Ross O'Hagan, ${ }^{3}$ Artine Arzani, ${ }^{6}$ Arsalan Haghdel, ${ }^{6}$ \\ Clarence C Lim, ${ }^{7}$ Vwaire Orhurhu, ${ }^{4,8}$ Ivan Urits, ${ }^{4,8}$ Anh L Ngo, ${ }^{4,9}$ \\ Benjamin S Glicksberg, ${ }^{3,10}$ Kathryn T Hall, ${ }^{11}$ Darshan Mehta, ${ }^{4,12,13}$ \\ Richard S Cooper, ${ }^{14}$ Girish N Nadkarni ${ }^{3,15}$
}

To cite: Paranjpe MD, Chin AC, Paranjpe I, et al. Self-reported health without clinically measurable benefits among adult users of multivitamin and multimineral supplements: a cross-sectional study. BMJ Open 2020;10:e039119. doi:10.1136/ bmjopen-2020-039119

- Prepublication history and additional material for this paper is available online. To view these files, please visit the journal online (http://dx.doi.org/10. 1136/bmjopen-2020-039119).

MDP and ACC contributed equally.

Received 06 April 2020 Revised 29 August 2020 Accepted 02 September 2020

D) Check for updates

(c) Author(s) (or their employer(s)) 2020. Re-use permitted under CC BY-NC. No commercial re-use. See rights and permissions. Published by BMJ.

For numbered affiliations see end of article.

Correspondence to Manish D Paranjpe; manish_paranjpe@hms. harvard.edu

\section{ABSTRACT}

Objective Multiple clinical trials fail to identify clinically measurable health benefits of daily multivitamin and multimineral (MVM) consumption in the general adult population. Understanding the determinants of widespread use of MVMs may guide efforts to better educate the public about effective nutritional practices. The objective of this study was to compare self-reported and clinically measurable health outcomes among MVM users and nonusers in a large, nationally representative adult civilian non-institutionalised population in the USA surveyed on the use of complementary health practices.

Design Cross-sectional analysis of the effect of MVM consumption on self-reported overall health and clinically measurable health outcomes.

Participants Adult MVM users and non-users from the 2012 National Health Interview Survey $(n=21603)$.

Primary and secondary outcome measures Five psychological, physical, and functional health outcomes: (1) self-rated health status, (2) needing help with routine needs, (3) history of 10 chronic diseases, (4) presence of 19 health conditions in the past 12 months, and (5) Kessler 6-Item (K6) Psychological Distress Scale to measure nonspecific psychological distress in the past month.

Results Among 4933 adult MVM users and 16670 adult non-users, MVM users self-reported $30 \%$ better overall health than non-users (adjusted OR 1.31; 95\% Cl 1.17 to 1.46; false discovery rate adjusted $p<0.001$ ). There were no differences between MVM users and non-users in history of 10 chronic diseases, number of present health conditions, severity of current psychological distress on the $\mathrm{K} 6 \mathrm{Scale}$ and rates of needing help with daily activities. No effect modification was observed after stratification by sex, education, and race.

Conclusions MVM users self-reported better overall health despite no apparent differences in clinically measurable health outcomes. These results suggest that widespread use of multivitamins in adults may be a result of individuals' positive expectation that multivitamin use leads to better health outcomes or a self-selection bias in which MVM users intrinsically harbour more positive views regarding their health.

\section{Strengths and limitations of the study}

- This study links better self-reported health, absence of clinically measurable benefits, and multivitamin and multimineral supplement use in the same population.

- Data are derived from a large, national survey across the USA.

- Results have broad implications for public health and the multibillion dollar supplement industry.

- Cross-sectional study design precludes the demonstration of a causal relationship between selfreported health and multivitamin and multimineral supplements.

- Self-reported health can be inherently biased and confounding.

\section{INTRODUCTION}

Consumption of multivitamins (MVs) and multiminerals (MMs) (together MVMs) as dietary supplements is widespread in the general US adult population, with some reports estimating $33 \%$ of Americans regularly take MVMs. ${ }^{1-4}$ While MVM supplementation is warranted for some individuals at high risk because of disease-related deficiency, ${ }^{5}$ the consumption of non-prescription, overthe-counter MVMs has not produced robust evidence for the wide-ranging health benefits expected by the general adult population. Likewise, large randomised clinical trials that evaluate MVMs at different doses, across both men and women at various ages, have failed to demonstrate benefit in prevention of chronic diseases. The Physicians' Health Study II (PHS II), a randomised placebocontrolled clinical trial of low-dose daily MVM use in older male physicians, found no reduction in major cardiovascular disease 
(CVD) events, myocardial infarction, stroke, and CVD mortality, ${ }^{6}$ and these results were independent of baseline nutritional status. ${ }^{7}$ A prospective cohort study of middle-aged and elderly women also indicated no effect of MVM use for the same CVD outcomes in PHS II. ${ }^{8}$ The SU.VI.MAX Study, a clinical trial of antioxidative MVMs in adults, found no effect on incidence of ischaemic CVD ${ }^{9}$ and high-dose MVMs did not reduce CVD events. ${ }^{10}$ Meta-analysis of these and other studies $(n=18)$ found no improvement in CVD outcomes in the general population. ${ }^{11}$ Based on these studies, the US Preventative Services Task Force does not recommend MVM use for the prevention of CVD. ${ }^{12} 13$

Data on the effect of MVM consumption on cognitive function in adults are also inconclusive. While results from PHS II found that long-term use of daily MVs did not provide cognitive benefits in men,${ }^{14}$ a meta-analysis on 10 studies concluded that MVs selectively enhanced free recall memory but no other cognitive functions. ${ }^{15}$ Intriguingly, 9 weeks of MVM use appears to improve multitasking and cognitive function during fatigue in women. ${ }^{16}$ With regard to cancer, PHS II demonstrated moderately reduced all-cancer risk in men consuming $\mathrm{MVs},{ }^{17}$ while data from the Women's Health Initiative Clinical Trials revealed no association. ${ }^{18}$ Some studies even link MVM use with increased cancer risk-a prospective cohort study of Swedish women found increased breast cancer risk associated with MVM use. ${ }^{19}$

The association of MVM use with all-cause mortality, like CVD, is null. While data from the Multiethnic Cohort Study cohort study indicated no association between MVM use and all-cause mortality, ${ }^{20}$ the Cancer Prevention Study (II) reported a 5\% higher rate of all-cause death among men using $\mathrm{MVs}^{21}$ and the Iowa Women's Health Study identified an association between MVM use and increased total mortality risk. ${ }^{22} \mathrm{~A}$ meta-analysis of these and other randomised trials $(n=21)$ demonstrated no effect of MVM use on mortality risk. ${ }^{23}$

While numerous reports on MVM consumption establish the lack of broad-spectrum, clinically measurable health benefits, the determinants of widespread MVM use by the general population are not well understood. The majority (52\%) of MVM users report using MVMs in an effort to prevent disease, which is even more puzzling in light of the paucity of randomised and observation data showing a positive health benefit of MVMs. ${ }^{24}$ Because nutritional supplements constitute a multibillion-dollar industry and can even be harmful when taken in excess, ${ }^{25}$ understanding the determinants of widespread MVM use has significant medical and financial consequences. Moreover, it is unclear whether MVM users, despite not being physiologically different from non-users, simply believe they are healthier. To address this question, we used data from the 2012 National Health Interview Survey ${ }^{26}$ (NHIS), which included a complementary and alternative (CAM) questionnaire comprising 21603 participants across the USA.

\section{METHODS}

\section{Data source}

All data were obtained from the 2012 National Health Interview Survey (NHIS), a nationally representative health survey conducted annually among civilian and non-institutionalised US participants by the Centers for Disease Control (CDC). All data were publicly available and did not require institutional review board approval. The 2012 NHIS was composed of a core questionnaire on health information administered to each selected household member. A randomly selected adult in each household was administered a more detailed health survey which included questions on access to care, specific health conditions and use of CAM (2012 only). In 2012, $77.6 \%$ of households completed the survey and $79.7 \%$ of adults selected completed the detailed survey. ${ }^{26}$

\section{Health status and health outcome measures}

We obtained data on adults (age $\geq 18$ years) derived from the Sample Adult Component who also participated in the Adult CAM File. This file surveys use of alternative medicines and therapies including daily MVM consumption, yoga and meditation. Consistent with previous NHIS studies ${ }^{27}$ we considered five psychological, physical,and functional health outcomes from questions in the Sample Adult Component: (1) self-rated health status (poor/fair vs excellent/very good/good), (2) needing help with routine needs such as eating (yes or no), (3) history of 10 chronic diseases (cancer, hypertension, coronary heart disease, stroke, chronic obstructive pulmonary disease, asthma, diabetes, arthritis, hepatitis, and weak/failing kidneys), (4) presence of 19 health conditions in the past 12 months (digestive, skin, and other allergy, acid reflux, hay fever, chest cold, nausea and vomiting, sore threat, infectious disease, recurring headache, memory loss, neurological problems, sprains, and abdominal, dental, muscle/bone, chronic, and skin pain), and (5) Kessler 6-Item (K6) Psychological Distress Scale ${ }^{28}$ score to measure non-specific psychological distress in the past month. Participants who refused to answer or did not know the answers to at least one of these questions were excluded from the study. Participants were classified as MVM users or non-users from their response to the question 'During the past 12 months, did take multi-vitamins or multi-minerals?' in the Adult CAM File. Participants who refused to answer or did not know their MVM use in the past 12 months were excluded from analyses.

\section{Statistical analysis}

For each outcome, the association between MVM use in the past year and health outcome was estimated using a logistic regression model adjusting for age, sex, race, region, education, income, employment status, health insurance status, presence of child in household, marital status, unmet medical care due to cost in the past year, and not seeing a health professional in office in the past 2 weeks. Multinomial logistic regression was used for outcomes with more than two levels (eg, number 
of chronic diseases, number of diseases in the past 12 months, Kessler-6 Item score). Binary logistic regression was used for outcomes with two levels (self-reported health and needing help with daily routines such as eating). Standard errors were estimated using weights provided by NHIS to account for the complex survey design and to produce nationally representative estimates. A multiple imputation strategy was used to estimate income in cases of missing responses to income as recommended by the National Centre for Health Statistics. ${ }^{29}$ All analyses were conducted using R (v3.5.1). p values were adjusted for multiple comparisons using a Benjamini-Hochberg procedure with false discovery rate (FDR) less than 0.01 deemed significant.

Stratified analyses were conducted in age (18-44, 45-64 and 65+years), race (white and non-white), sex (female and male), family income $(<100 \%, 100-199 \%, 200-299 \%$, $300-399 \%$ and $400 \%$ relative to the federal poverty level), and education level (did not graduate high school, high school graduate, college graduate or higher) groups to assess the association between MVM use and self-reported health in sociodemographic subgroups. In addition to stratified analyses, statistical interaction effects between MVM use and demographic variables (age, race, sex, family income, and education) on self-reported health were assessed using a multivariate regression model.

\section{Patients and public involvement}

Patients and the public were not involved in this study, including data collection, analysis and interpretation.

\section{RESULTS}

\section{Study cohort characteristics}

Sociodemographic differences between MVM users and non-users are presented in table 1. Our study included 4933 MVM users and 16670 non-users (table 1). As previously reported in data from the 2007-2010 and 2010-2014 National Health and Nutrition Examination Surveys (NHANES), ${ }^{30} 31$ compared with non-users, MVM users were significantly older, earned more income, were more likely to be female, more likely to be a college graduate, more likely to be married, and more likely to have health insurance. Unlike in previous studies, compared with MVM non-users, MVM users were less likely to be unemployed, to have a minor child in their household, and not to have an office visit for healthcare in the past 2 weeks (table 1). We observed no significant differences in percentage of non-English-speaking interviews and percentage having foregone medical care due to cost in the past year between MVM users and non-users (table 1).

Association between MVM usage and health status and health outcomes

Differences in health status and health outcomes between MVM users and non-users are displayed in table 2. Multivariate regression revealed that MVM users self-reported $30 \%$ better overall health than non-users (OR 1.31,
95\% CI 1.17 to 1.46 , FDR-adjusted $\mathrm{p}<0.001$; table 2 ). Strikingly, MVM users and non-users did not differ in history of 10 chronic disease (MVM users: mean 1.09 conditions, 95\% CI 1.06 to 1.11; non-users: mean 1.07, 95\% CI 1.03 to 1.11 ), number of present health conditions (MVM users: mean 2.7 conditions, $95 \%$ CI 2.7 to 2.8; non-users: mean $2.8,95 \%$ CI 2.7 to 2.9 ), severity of psychological distress on the K6 Scale (MVM users: mean K6 score 2.3, 95\% CI 2.3 to 2.4; non-users: mean $2.5,95 \%$ CI 2.4 to 2.6 ), and needing help with daily activities (OR $0.86,95 \%$ CI 0.71 to 1.04$)$.

Stratified analyses: association between MVM usage and selfreported overall health in sociodemographic subgroups

Table 3 reports the association between MVM usage and self-reported overall health in age, race, sex, income and education stratified subgroups (table 3). MVM use was associated with better self-reported health in the group aged $18-44$ years (OR 1.26 , 95\% CI 1.00 to 1.61 ) and 45-64years (OR 1.30, 95\% CI 1.08 to 1.57 ) and near significant among respondents aged 65 years and older (OR 1.20, 95\% CI 0.95 to 1.52, FDR-adjusted $\mathrm{p}$ value $=0.06)$ (table 3$)$. MVM use was associated with better self-reported health among white (OR 1.34, 95\% CI 1.07 to 1.67 ) and non-white (OR $1.26,95 \%$ CI 1.09 to 1.45) respondents (table 3). MVM use was associated with better self-reported health in both male (OR 1.33, 95\% CI 1.10 to 1.63 ) and female (OR 1.22, 95\% CI 1.05 to 1.41) respondents (table 3). Interestingly, MVM use was associated with better self-reported health in families with income less than $100 \%$ of the federal poverty level (FPL) (OR 1.42, 95\% CI 1.12 to 1.80 ), 100-199\% FPL (OR 1.37, 95\% CI 1.10 to 1.69 ) and 200-299\% FPL (OR 1.32, 95\% CI 1.01 to 1.72), but not in families whose income was 300-399\% FPL (OR 1.32, 95\% CI 0.88 to 1.98 ) or $400 \%$ FPL or higher (OR $1.15,95 \%$ CI 0.85 to 1.56) (table 3). MVM use was associated with better self-reported health in all education subgroups analysed, including respondents who did not complete high school (OR 1.38, 95\% CI 1.06 to 1.81), high school graduates (OR 1.21, 95\% CI 1.04 to 1.41 ) and college graduates (OR 1.37, 95\% CI 1.00 to 1.88 ) (table 3). All stratified analyses were conducted after adjusting for the potential confounding effects of age, sex, race, region, education, income, employment status, health insurance status, presence of child in household, marital status, unmet medical care due to cost in the past year, and not seeing a health professional in office in the past 2 weeks. The variable of stratification was not included as a covariate.

Statistical interaction effects between MVM use and demographic variables (age, race, family income and education) on self-reported overall health were assessed through a multivariate regression model in online supplemental table S1. We observed no significant association between MVM use and age, MVM use and race, MVM use and family income, and MVM use and education on selfreported overall income (online supplemental table S1). 
Table 1 Characteristics of American Adults by Multivitamin and Multimineral Supplement (MVM) Usage

\begin{tabular}{|c|c|c|c|}
\hline Characteristic & $\begin{array}{l}\text { MVM non-users } \\
\left(n=4933^{\star}\right)\end{array}$ & $\begin{array}{l}\text { MVM users } \\
\left(n=16670^{*}\right)\end{array}$ & FDR-adjusted $p$ value $†$ \\
\hline Weighted sample, \% & 22.4 (21.8 to 23.0) & 77.6 (76.9 to 78.0 ) & \\
\hline \multicolumn{4}{|l|}{ Age, \% (95\% Clł) } \\
\hline Mean age, years $(95 \% \mathrm{Cl})$ & 48.1 (47.4 to 48.7 ) & 49.7 (49.3 to 50.2 ) & $<0.001$ \\
\hline $18-27$ & 14.9 (13.8 to 16.2$)$ & $13.1(12.2$ to 14.1$)$ & \\
\hline $28-37$ & $16.6(15.4$ to 18.0$)$ & $16.9(16.2$ to 17.7$)$ & \\
\hline $48-57$ & 17.7 (16.4 to 19.0$)$ & 17.6 (16.9 to 18.3$)$ & \\
\hline $58-67$ & 14.3 (13.2 to 15.5$)$ & 15.4 (14.8 to 16.1$)$ & \\
\hline $68-80$ & 10.1 (9.2 to 11.1$)$ & $12.8(12.1$ to 13.5$)$ & \\
\hline$>80$ & 5.9 (5.1 to 6.8$)$ & 6.2 (5.7 to 6.7$)$ & \\
\hline
\end{tabular}

Race, \% (95\% Clł)

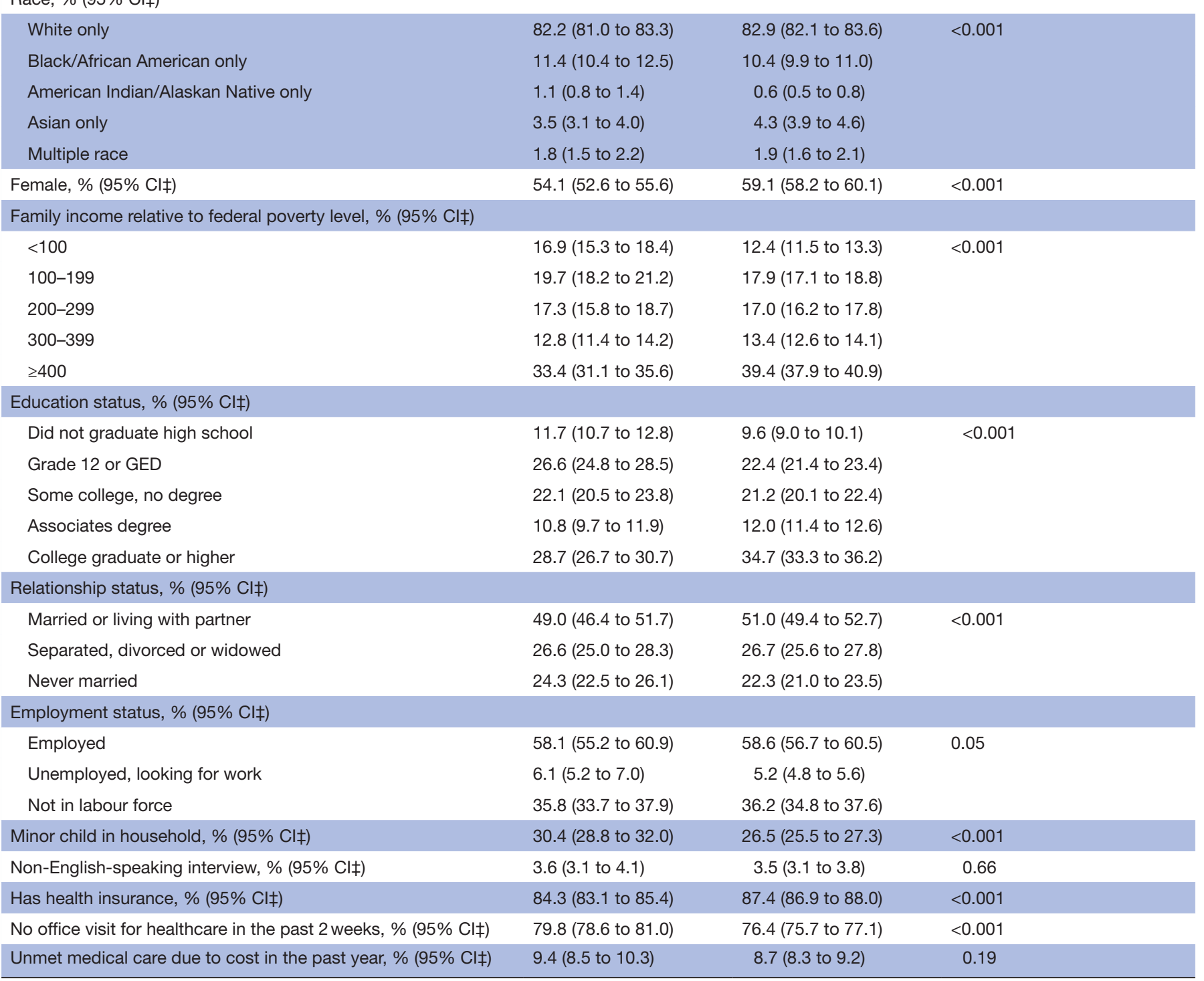

*Unweighted sample size.

TFDR-adjusted $\mathrm{p}$ value was computed using the Benjamini-Hochberg procedure; $\mathrm{p}$ values were computed using a two-sample t-test or $\chi^{2}$ test for independence. $\ddagger$ All confidence intervals were computed based on a Rao-Scott-scaled $\chi^{2}$ distribution for the loglikelihood from a binomial distribution using the Survey package in R.

§GED indicates completion of General Educational Development and provides certification of high-school level credentials in the United States.

FDR, false discovery rate; MVM, multivitamin and multimineral . 
Table 2 Association between MVM usage and health status

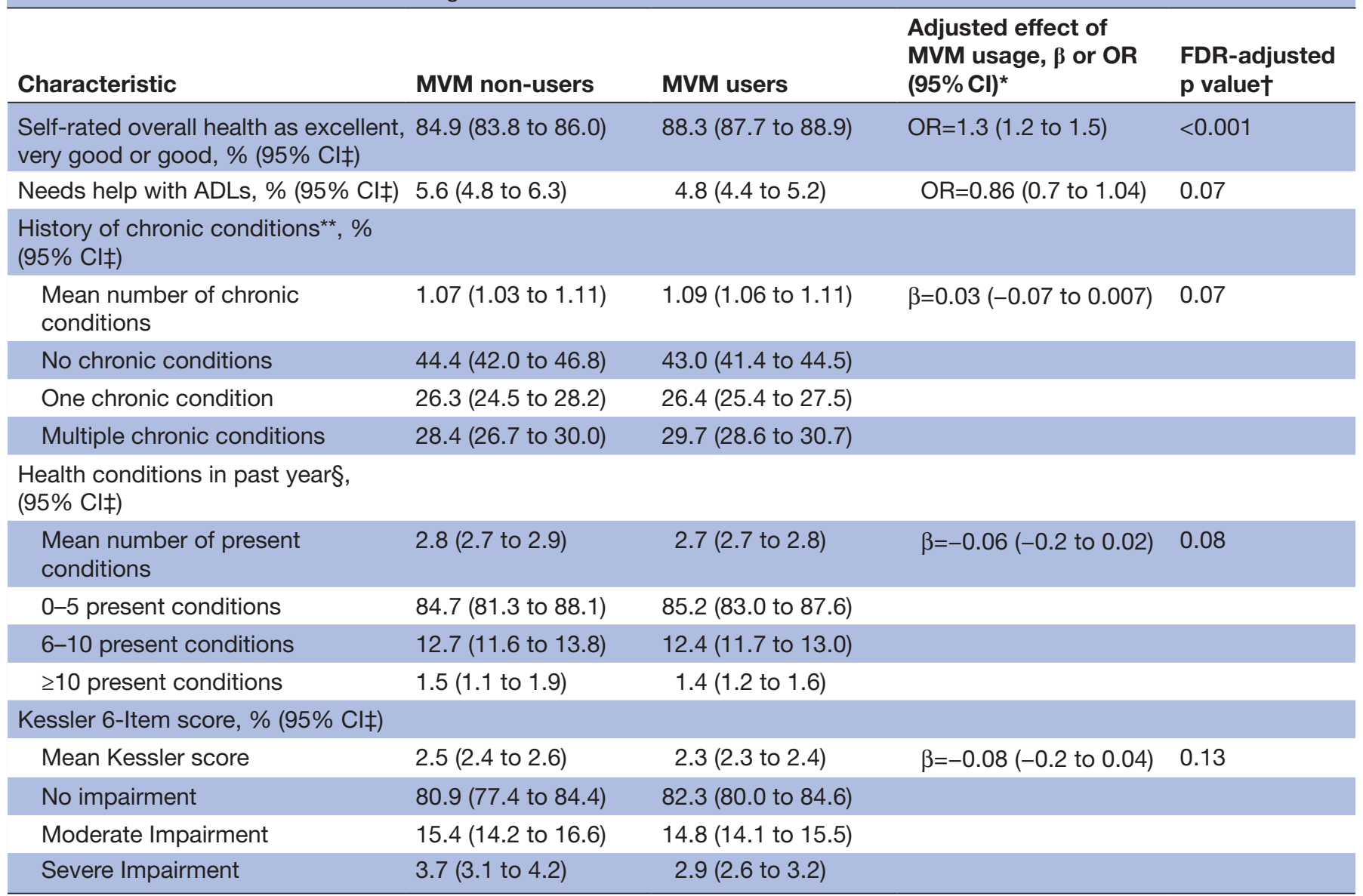

*Estimates were produced after adjusting for age, sex, race, region, education level, income, employment status, health insurance status, presence of child in household, marital status, unmet medical care due to cost in the past year, and not seeing a health professional in office in the past 2 weeks using a multivariate regression model.

†FDR-adjusted $\mathrm{p}$ values were computed using the Benjamini-Hochberg procedure.

$\ddagger$ All confidence intervals were computed based on a Rao-Scott-scaled $\chi^{2}$ distribution for the loglikelihood from a binomial distribution using the Survey package in R.

$\$ 19$ health conditions in the past 12 months included: respiratory, digestive, skin, and other allergy, acid reflux, hay fever, chest cold, nausea and vomiting, sore threat, infectious disease, recurring headache, memory loss, neurological problems, sprains, and abdominal, dental, muscle/bone, chronic, and skin pain.

IP value was defined using a multivariate regression model controlling for age, sex, race, region, education level, income, employment status, health insurance status, presence of child in household, marital status, unmet medical care due to cost in the past year, and not seeing a health professional in office in the past 2 weeks.

${ }^{\star \star} T e n$ chronic diseases included: cancer, hypertension, coronary heart disease, stroke, chronic obstructive pulmonary disease, asthma, diabetes, arthritis, hepatitis, and weak/failing kidneys.

$\mathrm{ADL}$, activities of daily living; FDR, false discovery rate; MVM, multivitamin and multimineral.

\section{DISCUSSION}

This present study is the first to simultaneously analyse the association between MVM use and both self-reported health and clinical health outcomes. In this work, we found that MVM users self-report $30 \%$ better overall health than non-users despite no clinically assessed differences in health. Our finding that MVM users and non-users do not differ in various psychological, physical and functional outcomes corroborates previous reports that MVMs do not improve overall health in the general adult population. ${ }^{5-22}$ Our stratified analysis revealed that MVM use is associated with better self-reported overall health across all race, sex and education groups, and in individuals under 65 and with family incomes below $300 \%$ FPL. The lack of association between MVM usage and self-reported health in individuals with family income greater than $300 \%$ FPL may be related to sample size and should be replicated in a follow-up study. Taken together, these findings help elucidate explanations underlying widespread MVM usage despite no generalised clinical benefits.

The results here suggest two potential explanations underlying widespread MVM consumption in the absence of clinically measurable benefits: MVM users believe in the efficacy of MVMs by harbouring a positive expectation regarding the health benefits of MVMs; and MVM 
Table 3 Association between MVM usage and self-reported overall health in sociodemographic subgroups

\begin{tabular}{|c|c|c|c|c|}
\hline Group & $\begin{array}{l}\text { Self-rated overall } \\
\text { health as excellent, } \\
\text { very good or good, } \\
\%\left(95 \% \mathrm{CI}^{\star}\right) \text {, } \\
\text { MVM Non-Users }\end{array}$ & $\begin{array}{l}\text { Self-rated overall } \\
\text { health as excellent, } \\
\text { very good or good, } \\
\%\left(95 \% \mathrm{CI}^{\star}\right), \text { MVM } \\
\text { Users }\end{array}$ & $\begin{array}{l}\text { Adjusted effect } \\
\text { of MVM usage } \\
\text { on self-reported } \\
\text { health, OR }(95 \% \\
\left.\mathrm{Cl}^{\star}\right) \dagger\end{array}$ & $\begin{array}{l}\text { FDR } \\
\text { adjusted } p \\
\text { value }\end{array}$ \\
\hline \multicolumn{5}{|l|}{ Age, years } \\
\hline $18-44$ & 92.3 (91.1 to 93.5$)$ & 94.2 (93.6 to 94.8$)$ & $1.3(1.0$ to 1.6$)$ & 0.03 \\
\hline $45-64$ & 79.9 (77.8 to 82.1$)$ & 85.3 (84.2 to 86.4 ) & $1.3(1.1$ to 1.6$)$ & 0.009 \\
\hline$\geq 65$ & 77.2 (73.8 to 80.5$)$ & 82.0 (80.6 to 83.4$)$ & $1.2(1.0$ to 1.5$)$ & 0.06 \\
\hline \multicolumn{5}{|l|}{ Race } \\
\hline White & 85.9 (84.7 to 87.2 ) & 89.1 (88.5 to 89.7 ) & $1.3(1.1$ to 1.7$)$ & 0.009 \\
\hline Non-white & 80.0 (77.2 to 82.7$)$ & 84.2 (82.8 to 85.6$)$ & $1.3(1.1$ to 1.5$)$ & 0.007 \\
\hline \multicolumn{5}{|l|}{ Sex } \\
\hline Female & 84.0 (82.5 to 85.4$)$ & 88.1 (87.4 to 88.9$)$ & $1.2(1.1$ to 1.4$)$ & 0.009 \\
\hline Male & 85.9 (84.2 to 87.7$)$ & 88.4 (87.5 to 89.3$)$ & $1.3(1.1$ to 1.6$)$ & 0.009 \\
\hline \multicolumn{5}{|c|}{$\begin{array}{l}\text { Family income relative to federal poverty level, } \\
\%(95 \% \mathrm{Cl})\end{array}$} \\
\hline$<100$ & 71.7 (68.0 to 75.4$)$ & 75.6 (73.1 to 78.1$)$ & $1.4(1.1$ to 1.8$)$ & 0.007 \\
\hline $100-199$ & 76.4 (73.6 to 79.2$)$ & 80.7 (79.0 to 82.4$)$ & $1.4(1.1$ to 1.7$)$ & 0.007 \\
\hline $200-299$ & $84.8(82.1$ to 87.5$)$ & 87.3 (85.9 to 88.6$)$ & $1.3(1.0$ to 1.7$)$ & 0.04 \\
\hline 300-399 & 89.6 (86.4 to 92.7$)$ & 91.0 (89.6 to 92.4) & 1.3 (0.9 to 2.0$)$ & 0.15 \\
\hline$\geq 400$ & 94.8 (93.5 to 96.1$)$ & 95.2 (94.6 to 95.8$)$ & $1.1(0.8$ to 1.6$)$ & 0.23 \\
\hline \multicolumn{5}{|l|}{ Education } \\
\hline Did not graduate high school & $67.2(63.1$ to 71.3$)$ & 71.9 (69.7 to 74.2$)$ & $1.4(1.1$ to 1.9$)$ & 0.01 \\
\hline High school graduate & 84.1 (82.6 to 85.5$)$ & 86.7 (85.9 to 87.4$)$ & $1.2(1.0$ to 1.4$)$ & 0.01 \\
\hline College graduate or higher & 93.8 (92.4 to 95.1$)$ & 95.3 (94.7 to 95.9$)$ & 1.4 (1.0 to 1.9$)$ & 0.03 \\
\hline
\end{tabular}

*All confidence intervals were computed based on a Rao-Scott-scaled $\chi^{2}$ distribution for the loglikelihood from a binomial distribution using the Survey package in $\mathrm{R}$.

†Estimates were produced after adjusting for age, sex, race, region, education level, income, employment status, health insurance status, presence of child in household, marital status, unmet medical care due to cost in the past year, and not seeing a health professional in office in the past 2 weeks.

†FDR-adjusted $p$ values were computed using the Benjamini-Hochberg procedure; $p$ value was defined using a multivariate regression model controlling for age, sex, race, region, education level, income, employment status, health insurance status, presence of child in household, marital status, unmet medical care due to cost in the past year, and not seeing a health professional in office in the past 2 weeks.

FDR, false discovery rate; MVM, multivitamin and multimineral.

users intrinsically harbour a more positive outlook on their personal health regardless of MVM usage. A growing body of evidence suggests that positive expectation influences treatment outcomes for diseases including heart disease, ${ }^{32-35}$ cancer, ${ }^{36} 37$ musculoskeletal disorders, ${ }^{38} 39$ injuries $^{4041}$ and obesity. ${ }^{42-44}$ Under a positive expectation model, MVM users are more likely to harbour a positive expectation regarding the clinical efficacy of MVMs and thus are more likely to self-report as having excellent or good overall health. In the case of MVM usage, it is interesting that the presence of positive expectations did not influence clinically measurable health outcomes, unlike in other treatments. The effect of positive expectations in the MVM user community is made even stronger when one considers that the majority of MVM and supplements are sold to the so-called 'worried-well' population ${ }^{45}$ who may assign greater weight to the purported health benefits of dietary supplements and alternative therapies. It is possible that members of this population are more susceptible to positive expectations and may thereby continue to use MVMs in the absence of clinical benefits.

The second mechanism, in which MVM users intrinsically harbour greater positive views about their health, may be explained in part by certain combinations of sociodemographic determinants that influence selfreported health. While age, sex, income, education and location of residence have been previously shown to affect self-reported health in diverse populations,${ }^{46-48}$ combinations of other characteristics may also cause MVM users to harbour intrinsically more positive views regarding their health in the absence of clinical differences. Further research is necessary to elucidate these characteristics.

Our results are consistent with existing work from two studies: the first being a 2013 study involving 11956 
adults from the 2007-2010 NHANES that demonstrated MVM users exhibit better self-reported health than nonusers $^{31}$; and second, a 2014 study involving 5536 Coast Guard and military personnel which found that MVM users were significantly more likely to self-report their general health as excellent or good. ${ }^{49}$ While informative, these previous studies only focused on self-reported health as an outcome. In the present study, we considered self-reported health in addition to clinically measurable health outcomes. This is an important distinction establishing that MVM users experience better self-reported health in the absence of clinically measurable health improvement. Nevertheless, it is encouraging that our results are consistent across the NHANES, military and Coast Guard, and NHIS study cohorts, and robust to different statistical analysis methodologies.

Limitations of this study include the cross-sectional design, reliability of self-reported health within NHIS, income estimation using multiple imputation, indication bias and non-response bias. First, the cross-sectional study design prevents demonstration of a causal relationship between MVM use and self-reported health. The lack of longitudinal data available to assess changes in selfreported health before and after MVM supplementation prevents us from differentiating the two aforementioned explanations that may contribute to widespread MVM use. Second, self-reported health within the NHIS may inherently harbour reporting bias and residual confounding. In addition to reporting bias and residual confounding, a self-reported binary response to the question of whether one has taken MVMs in the past 12 months precludes any analysis of the dose-dependent effects of MVMs in our cohort. This is especially important considering some vitamins and minerals have known U-shaped associations with disease, where disease risk is elevated at both high and low vitamin and mineral levels. ${ }^{50-53}$ Further, use of both multivitamins and multiminerals were asked together as part of the same question in the NHIS questionnaire. This prevented us from analysing multivitamin and multimineral effects in isolation. Moreover, different MVM preparations can differ in their nutritional composition, quality and bioavailability. Some individuals may take multiple MVMs whose constituents could interact with each other. Because the brand of multivitamin being taken was not asked of MVM users in NHIS, we could not identify differences in nutritional composition, quality, bioavailability and chemical interaction that may be driving the results in this study.

Third, despite being recommended by the NHIS, ${ }^{29}$ the multiple imputation technique used to calculate income in cases in which data were missing may generate estimation errors. Another limitation to the income-stratified results for self-reported overall health may stem from the inability to take account of income mobility. Interestingly, it has been previously demonstrated that while high incomes are associated with longer life expectancies, accounting for income mobility reduces the gap by approximately $50 \% .^{54}$
A portion of our cohort may have been prescribed MVMs, specific vitamins or specific minerals for indications including micronutrient deficiency, pregnancy, iron deficiency anaemia, osteoporosis, Crohn's disease and others, thereby contributing to indication bias. ${ }^{55-60}$ Previous estimates have suggested approximately $1 \%$ of physician office visits in the USA include a prescription or recommendation for MVMs. ${ }^{61}$ One can imagine a scenario in which MVM users and non-users are imbalanced in the proportion of medical cases that require MVM supplementation (ie, micronutrient deficiency or pregnancy). In such a scenario, it may falsely appear that MVM use is not associated with clinical benefits. In the present study, owing to a lack of information regarding the reason for taking MVMs, we were unable to fully account for indication bias present in our cohort.

In addition to indication bias, the NHIS, like other surveys, is known to suffer from non-response bias ${ }^{62}$ For example, a previous study found that the 1990-2009 NHIS population had an approximately $14 \%$ lower mortality than the general population. ${ }^{62}$ Post hoc methods to address non-response bias include creating sample weights based on demographic variables and selection probabilities, as was used in the present study. However, survey weighting, while a standard practice, may not fully account for non-response bias, especially if the survey weights do not take into account common differences between survey responders and non-responders, such as smoking and alcohol use. ${ }^{63}$ As a result, non-response bias may limit the generalisability of our results to the broader population.

\section{CONCLUSIONS}

Using nationally representative survey data on health outcomes, our study reveals that MVM users self-report better overall health than non-users despite not exhibiting improved health by clinically measurable standards. Furthermore, we identify specific sociodemographic subgroups of MVM users that are more prone to this behaviour. The multibillion-dollar nature of the nutritional supplement industry means that understanding the determinants of widespread MVM use has significant medical and financial consequences. Our findings suggest that widespread use of multivitamins in adults may be a result of individuals' positive expectation that multivitamin use leads to better health outcomes or a selfselection bias in which MVM users intrinsically harbour more positive views regarding their health.

\section{Author affiliations}

${ }^{1}$ Health Sciences and Technology, Harvard Medical School, Boston, Massachusetts, USA

${ }^{2}$ Weill Cornell/Rockefeller/Sloan Kettering Tri-Institutional MD-PhD Program, New York, NY, USA

${ }^{3}$ Hasso Plattner Institute for Digital Health at Mount Sinai, Icahn School of Medicine at Mount Sinai, New York, New York, USA

${ }^{4}$ Harvard Medical School, Boston, Massachusetts, USA 
${ }^{5}$ Medical Scientist Training Program, Yale University School of Medicine, New Haven, Connecticut, USA

${ }^{6}$ Weill Cornell Medical College, New York City, New York, USA

${ }^{7}$ Texas A\&M University System Health Science Center College of Medicine, Bryan, Texas, USA

${ }^{8}$ Department of Anesthesia, Critical Care and Pain Medicine, Pain Division,

Massachusetts General Hospital, Boston, Massachusetts, USA

${ }^{9}$ Department of Anesthesia, Critical Care, and Pain Medicine, Beth Israel Deaconess

Medical Center, Boston, Massachusetts, USA

${ }^{10}$ Department of Genetics and Genomic Sciences, Icahn School of Medicine at Mount Sinai, New York, NY, United States

${ }^{11}$ Division of Preventive Medicine, Brigham and Women's Hospital, Boston,

Massachusetts, USA

${ }^{12}$ Benson-Henry Institute for Mind Body Medicine, Massachusetts General Hospital, Boston, Massachusetts, USA

${ }^{13}$ Osher Center for Integrative Medicine, Brigham and Women's Hospital, Boston,

Massachusetts, United States

${ }^{14}$ Loyola University Medical Center, Maywood, Illinois, USA

${ }^{15}$ Icahn School of Medicine at Mount Sinai, New York City, New York, USA

Twitter Manish D Paranjpe @mparanjpe96, Alfred C Chin @alfredcchin and Benjamin S Glicksberg @BenGlicksberg

Contributors MDP and ACC conceived and designed the study. MDP extracted data from NHANES. MDP, ACC, IP, PQD, JKW, RO, NJR, AA, AH, CCL, VO, IU, ALN, BSG, KTH, DHM and GNN analysed the data. MDP, ACC, KTH and DHM wrote the manuscript. MDP, ACC, KTH, DHM, GNN and RSC critically revised the manuscript for important intellectual content. All authors commented and approved the manuscript.

Funding ACC and PQD were supported by NIH Medical Scientist Training Program Training Grants T32GM007739 and T32GM007205 respectively.

Competing interests None declared.

Patient consent for publication Not required.

Provenance and peer review Not commissioned; externally peer reviewed.

Data availability statement Data are available in a public, open access repository. All data used in the study are publicly available from the National Health Interview Survey.

Open access This is an open access article distributed in accordance with the Creative Commons Attribution Non Commercial (CC BY-NC 4.0) license, which permits others to distribute, remix, adapt, build upon this work non-commercially, and license their derivative works on different terms, provided the original work is properly cited, appropriate credit is given, any changes made indicated, and the use is non-commercial. See: http://creativecommons.org/licenses/by-nc/4.0/.

ORCID iD

Manish D Paranjpe http://orcid.org/0000-0002-6463-8965

\section{REFERENCES}

1 Kantor ED, Rehm CD, Du M, et al. Trends in dietary supplement use among US adults from 1999-2012. JAMA 2016;316:1464.

2 Bailey RL, Gahche JJ, Lentino CV, et al. Dietary supplement use in the United States, 2003-2006. J Nutr 2011;141:261-6.

3 Radimer K, Bindewald B, Hughes J, et al. Dietary supplement use by US adults: data from the National health and nutrition examination survey, 1999-2000. Am J Epidemiol 2004;160:339-49.

4 Gahche J, Bailey R, Burt V, et al. Dietary supplement use among U.S. adults has increased since NHANES III (1988-1994). NCHS Data Brief 2011:1-8

5 Manson JE, Bassuk SS. Vitamin and mineral supplements: what clinicians need to know. JAMA 2018;319:859-860.

6 Sesso HD, Christen WG, Bubes V, et al. Multivitamins in the prevention of cardiovascular disease in men: the physicians' health study II randomized controlled trial. JAMA 2012;308:1751-60.

7 Rautiainen S, Gaziano JM, Christen WG, et al. Effect of baseline nutritional status on long-term multivitamin use and cardiovascular disease risk. JAMA Cardiol 2017;2:617.

8 Rautiainen S, Lee I-M, Rist PM, et al. Multivitamin use and cardiovascular disease in a prospective study of women. Am J Clin Nutr 2015;101:144-52.

9 Hercberg S, Galan P, Preziosi P, et al. The SU.VI.MAX study: a randomized, placebo-controlled trial of the health effects of antioxidant vitamins and minerals. Arch Intern Med 2004;164:2335-42

10 Lamas GA, Boineau R, Goertz C, et al. Oral high-dose multivitamins and minerals after myocardial infarction: a randomized trial. Ann Intern Med 2013;159:797.

$11 \mathrm{Kim}$ J, Choi J, Kwon SY, et al. Association of multivitamin and mineral supplementation and risk of cardiovascular disease: a systematic review and meta-analysis. Circ Cardiovasc Qual Outcomes 2018;11:e004224.

12 Moyer VA, U.S. Preventive Services Task Force. Vitamin, mineral, and multivitamin supplements for the primary prevention of cardiovascular disease and cancer: U.S. preventive services Task force recommendation statement. Ann Intern Med 2014;160:558-64.

13 Fortmann SP, Burda BU, Senger CA, et al. Vitamin, mineral, and multivitamin supplements for the primary prevention of cardiovascular disease and cancer: a systematic evidence review for the US. preventive services Task force. Evid Rep 2013.

14 Grodstein F, O'Brien J, Kang JH, et al. Long-Term multivitamin supplementation and cognitive function in men: a randomized trial. Ann Intern Med 2013;159:806-14.

15 Grima NA, Pase MP, Macpherson $\mathrm{H}$, et al. The effects of multivitamins on cognitive performance: a systematic review and meta-analysis. J Alzheimers Dis 2012;29:561-9.

16 Haskell CF, Robertson B, Jones E, et al. Effects of a multi-vitamin/ mineral supplement on cognitive function and fatigue during extended multi-tasking. Hum Psychopharmacol 2010;25:448-61.

17 Gaziano JM, Sesso HD, Christen WG, et al. Multivitamins in the prevention of cancer in men: the physicians' health study II randomized controlled trial. JAMA 2012;308:1871-80.

18 Neuhouser ML, Wassertheil-Smoller S, Thomson C, et al. Multivitamin use and risk of cancer and cardiovascular disease in the women's health Initiative cohorts. Arch Intern Med 2009;169:294.

19 Larsson SC, Åkesson A, Bergkvist L, et al. Multivitamin use and breast cancer incidence in a prospective cohort of Swedish women. Am J Clin Nutr 2010;91:1268-72.

20 Park S-Y, Murphy SP, Wilkens LR, et al. Multivitamin use and the risk of mortality and cancer incidence: the Multiethnic cohort study. Am J Epidemiol 2011;173:906-14.

21 Watkins ML, Erickson JD, Thun MJ, et al. Multivitamin use and mortality in a large prospective study. Am J Epidemiol 2000;152:149-62.

22 Mursu J, Robien K, Harnack LJ, et al. Dietary supplements and mortality rate in older women: the lowa women's health study. Arch Intern Med 2011;171:1625-33.

23 Macpherson H, Pipingas A, Pase MP. Multivitamin-multiminera supplementation and mortality: a meta-analysis of randomized controlled trials. Am J Clin Nutr 2013;97:437-44.

24 McGinnis JM, Birt DF, Brannon PM, et al. National Institutes of health State-of-the-science conference statement: multivitamin/mineral supplements and chronic disease prevention. Ann Intern Med 2006;145:364-71.

25 Geller Al, Shehab N, Weidle NJ, et al. Emergency department visits for adverse events related to dietary supplements. $N$ Engl J Med 2015;373:1531-40.

262012 NHIS survey description. Available: https://ftp.cdc.gov/pub/ health_statistics/nchs/dataset_documentation/NHIS/2012/srvydesc. pdf [Accessed 24 Apr 2019].

27 Gonzales G, Przedworski J, Henning-Smith C. Comparison of health and health risk factors between lesbian, gay, and bisexual adults and heterosexual adults in the United States: results from the National health interview survey. JAMA Intern Med 2016;176:1344-51.

28 Kessler RC, Barker PR, Colpe LJ, et al. Screening for serious mental illness in the general population. Arch Gen Psychiatry 2003;60:184.

29 Center for Health Statistics - Division of Health Interview Statistics N. Multiple imputation of family income and personal earnings in the National health interview survey: methods and examples, 2013. Available: https://www.cdc.gov/nchs/data/nhis/tecdoc13.pdf [Accessed 8 Jul 2019].

30 Cowan A, Jun S, Gahche J, et al. Dietary supplement use differs by socioeconomic and health-related characteristics among U.S. adults, NHANES 2011-2014. Nutrients 2018;10:1114.

31 Bailey RL, Gahche JJ, Miller PE, et al. Why US adults use dietary supplements. JAMA Intern Med 2013;173:355.

32 Petrie KJ, Weinman J, Sharpe N, et al. Role of patients' view of their illness in predicting return to work and functioning after myocardial infarction: longitudinal study. BMJ 1996;312:1191-4.

33 Juergens MC, Seekatz B, Moosdorf RG, et al. Illness beliefs before cardiac surgery predict disability, quality of life, and depression 3 months later. J Psychosom Res 2010;68:553-60. 
34 Barefoot JC, Brummett BH, Williams RB, et al. Recovery expectations and long-term prognosis of patients with coronary heart disease. Arch Intern Med 2011;171:929-35.

35 Habibović M, Pedersen SS, van den Broek KC, et al. Monitoring treatment expectations in patients with an implantable cardioverterdefibrillator using the EXPECT-ICD scale. Europace 2014;16:1022-7.

36 Colagiuri B, Zachariae R. Patient expectancy and postchemotherapy nausea: a meta-analysis. Ann Behav Med 2010;40:3-14.

37 Nestoriuc Y, von Blanckenburg P, Schuricht F, et al. Is it best to expect the worst? influence of patients' side-effect expectations on endocrine treatment outcome in a 2-year prospective clinical cohort study. Ann Oncol 2016;27:1909-15.

38 Mahomed NN, Liang MH, Cook EF, et al. The importance of patient expectations in predicting functional outcomes after total joint arthroplasty. J Rheumatol 2002;29:1273-9.

39 Oettingen G, Mayer D. The motivating function of thinking about the future: expectations versus fantasies. J Pers Soc Psychol 2002;83:1198-212.

40 Booth-Kewley S, Schmied EA, Highfill-McRoy RM, et al. A prospective study of factors affecting recovery from musculoskeletal injuries. J Occup Rehabil 2014;24:287-96.

41 Murgatroyd DF, Harris IA, Tran Y, et al. Predictors of return to work following motor vehicle related orthopaedic trauma. BMC Musculoskelet Disord 2016;17:171.

42 Oettingen G, Wadden TA. Expectation, fantasy, and weight loss: is the impact of positive thinking always positive? Cognit Ther Res 1991.

43 Armitage CJ, Norman P, Alganem S, et al. Expectations are more predictive of behavior than behavioral intentions: evidence from two prospective studies. Ann Behav Med 2015;49:239-46.

44 Crane MM, Ward DS, Lutes LD, et al. Theoretical and behavioral mediators of a weight loss intervention for men. Ann Behav Med 2016:50:460-70.

45 Lentjes MAH. The balance between food and dietary supplements in the general population. Proc Nutr Soc 2019;78:97-109.

46 Boerma T, Hosseinpoor AR, Verdes E, et al. A global assessment of the gender gap in self-reported health with survey data from 59 countries. BMC Public Health 2016;16:675.

47 Hosseinpoor AR, Stewart Williams J, Amin A, et al. Social determinants of self-reported health in women and men: understanding the role of gender in population health. PLOS One 2012; 7:e34799.

48 Bethune R, Absher N, Obiagwu M, et al. Social determinants of self-reported health for Canada's Indigenous peoples: a public health approach. Public Health 2019;176:172-80.

49 Austin KG, McGraw SM, Lieberman HR. Multivitamin and protein supplement use is associated with positive mood states and health behaviors in US military and coast guard personnel. $J$ Clin Psychopharmacol 2014;34:595-601.

50 Bleicher K, Cumming RG, Naganathan V, et al. U-Shaped association between serum 25-hydroxyvitamin $D$ and fracture risk in older men: results from the prospective population-based CHAMP study. $J$ Bone Miner Res 2014;29:2024-31.

51 Hayes DP. Adverse effects of nutritional inadequacy and excess: a hormetic model. Am J Clin Nutr 2008;88:578S-81.

52 Calabrese EJ, Baldwin LA. U-Shaped dose-responses in biology, toxicology, and public health. Annu Rev Public Health 2001;22:15-33.

53 Aleksova A, Beltrami AP, Belfiore R, et al. U-Shaped relationship between vitamin $D$ levels and long-term outcome in large cohort of survivors of acute myocardial infarction. Int $J$ Cardiol 2016;223:962-6.

54 Kreiner CT, Nielsen TH, Serena BL. Role of income mobility for the measurement of inequality in life expectancy. Proc Natl Acad Sci U S A 2018:115:11754-9.

55 Kamangar F, Emadi A. Vitamin and mineral supplements: do we really need them? Int J Prev Med 2012;3:221-6.

56 PrescQIPP. The prescribing of vitamins and minerals including vitamin B preparations (DROP-List) Nutrition-Support-in-Adults B107. vitamins and minerals (DROP-List) 2.1, 2015. Available: http:// pathways.nice.org.uk/pathways/

57 Fletcher J, Cooper SC, Ghosh S, et al. The role of vitamin D in inflammatory bowel disease: mechanism to management. Nutrients 2019;11:1019.

58 Alleyne M, Horne MK, Miller JL. Individualized treatment for irondeficiency anemia in adults. Am J Med 2008;121:943-8.

59 Blumberg J, Frei B, Fulgoni V, et al. Impact of frequency of MultiVitamin/Multi-Mineral supplement intake on nutritional adequacy and nutrient deficiencies in U.S. adults. Nutrients 2017;9:849.

60 Blumberg JB, Cena H, Barr SI, et al. The use of Multivitamin/ Multimineral supplements: a modified Delphi consensus panel report. Clin Ther 2018;40:640-57.

61 jeffery S, Muncie HL, Koch H. Prescription and recommendation of multivitamins by physicians in office based ambulatory care in the United States. Nutr Res 1988.

62 Keyes KM, Rutherford C, Popham F, et al. How healthy are survey Respondents compared with the general population?: using Surveylinked death records to compare mortality outcomes. Epidemiology 2018;29:299-307.

63 Gorman E, Leyland AH, McCartney G, et al. Assessing the representativeness of population-sampled health surveys through linkage to administrative data on alcohol-related outcomes. $A m \mathrm{~J}$ Epidemiol 2014;180:941-8. 